\title{
Energy-harvesting and energy aware routing algorithm for heterogeneous energy WSNs
}

\author{
Mohammed Mehdi Saleh ${ }^{1}$, Ruslan Saad Abdulrahman², Aymen Jaber Salman ${ }^{3}$ \\ ${ }^{1}$ Education College-Qaim, University of Anbar, Ramadi, Iraq \\ ${ }^{2,3}$ Department of Computer Engineering, College of Engineering, Al-Nahrain University, Baghdad, Iraq
}

\begin{tabular}{l}
\hline Article Info \\
\hline Article history: \\
Received Jul 14, 2021 \\
Revised Sep 8, 2021 \\
Accepted Sep 14, 2021 \\
\hline
\end{tabular}

Keywords:

Clustering

Heterogeneous networks

Sleep-awake mechanism

Solar energy harvesting

Wireless sensor networks

\begin{abstract}
Wireless sensor networks are regarded as the most essential components of contemporary technologies since they are in charge of sensing and monitoring processes, which are the primary functions of these technologies. Because these nodes rely on an unchangeable battery and are randomly deployed in the environment, node energy management is the most essential issue to consider when designing algorithms to enhance the network's life. Clustering is a wireless sensor network (WSN) routing technique that has been implemented in order to extend network lifetime. Also, it is trendy to increase the energy levels of the node battery by utilizing various energy harvesting techniques in order to extend the network lifetime. In this paper, a new energy-aware clustering algorithm (EHEARA) has been proposed. The proposed algorithm is based on a dynamic clustering function and adopts a solar energy harvesting scheme in order to improve network lifetime. Furthermore, the active-sleep mechanism was used to distribute node activity and balance communication among nodes within clusters and cluster heads with the base station. The proposed algorithm is simulated using matrix laboratory (MATLAB), and the results show that it outperforms the low energy adaptive clustering hierarchy (LEACH), distributed energy efficient clustering (DEEC), and stable election protocol (SEP) algorithms in terms of network lifetime, energy consumption, and network throughput.
\end{abstract}

This is an open access article under the CC BY-SA license.

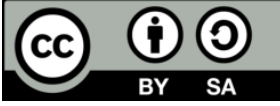

\section{Corresponding Author:}

Mohammed Mehdi Saleh

Education College-Qaim

University of Anbar, 11, Ramadi, Iraq

Email: mohammedmhedi@uoanbar.edu.iq

\section{INTRODUCTION}

Recently, wireless sensor networks (WSNs) are becoming increasingly important in many fields, such military, industrial control, environmental monitoring, home automation. This type of network is mainly composed of hundreds of thousands of nodes with limited resources known as sensors [1], [2]. These sensors have the ability to communicate with each other to monitor and detect different parameters for a particular application. The sensors will send their sensed data which are continuously collected at all or at some of the sensor nodes and sent to the base station (BS) for further processing [3]. Generally, sensors are typically powered by a battery, so as sensor networks grow in number and size, the replacement of exhausted batteries becomes time-consuming and inefficient [4]. While getting WSN big attention in the last decade, power consumption in the sending and receiving data between nodes is considered as one of the main challenges that needs more and more research from the scientific community [5]. In addition, it may be deployed in unreachable environments, making it difficult to manage energy consumption during the sensing and routing process. In all cases, one of the major goals for the design and development of any application in WSN is to 
maintain the sensor nodes alive and usable for as long as needed [6]-[8]. There are various fields wherein the energy of sensor nodes consumed such as communication, sensing, processing, idleness and sleeping. Therefore, by managing these fields, the power of the nodes can be controlled [9]-[11]. The most critical problem is how the sensor nodes transmit their collected data to the BS. Heterogeneity is another challenge that should be considered, which can occur at different levels, for example, sensor nodes hardware capabilities, energy levels, communication links, sensing skills and tasks, and energy harvesting techniques [12]-[14].

The research community has proposed many routing algorithms to cope with these challenges, where cluster topology is one of these proposals and is considered as the most used because of its efficiency in terms of energy consumption [15]-[17]. Based on research in this area, the hierarchical routing technique is more efficient in terms of minimizing energy consumption and prolonging the life of the network [18]. In this technique, the nodes are organized into clusters, and there is a central node called the cluster head $(\mathrm{CH})$ for each cluster. CHs will be responsible for collecting data from sensors within the cluster, and then forwarding it to the BS. By managing and controlling the clustering process and the selection role of CHs, the energy consumption of the nodes can be balanced and reduced [19]. Furthermore, in order to make the process more efficient, the wake-up and sleeping mechanism of the nodes can be implemented on the basis of certain conditions and techniques. Instantly, once the residual energy of the node is below a certain threshold, it will send it to sleep mode where the consumption is too small, it can be ignored [20]. Solar energy harvesting (SEH) is a new technology used in WSN nodes as a solution to energy constraints, converting solar light into electrical energy to recharge node battery [21], [22]. The energy harvested is also used for powering the WSN node directly. Solar energy is the most efficient and highly motivating factor in the design of WSN nodes compared to other energy sources such as kinetics, thermal and radio frequency (RF) energy. The average potential energy collected from outdoor solar energy is $10-15 \mathrm{~mW} / \mathrm{cm} 2$ with an efficiency of up to $30 \%[23]-[25]$.

A new energy harvesting routing algorithm for heterogeneous energy WSNs is proposed in this paper. The proposed algorithm takes into account the heterogeneity of the network, where nodes have varying energy levels, and the ability of nodes to harvest energy. In addition, the energy harvesting capabilities of the nodes are taken into account in this proposed algorithm in both cluster head $(\mathrm{CH})$ mode and cluster member (CM) mode. The network will be divided into two main groups according to energy level: the $\mathrm{CH}$ group and the $\mathrm{CM}$ group. In addition, a new approach was introduced to regulate the state of the nodes from active to sleep, and vice versa. Depending on what is given, we have chosen solar energy harvesting to provide alternative energy to WSNs as it has the highest power density and efficiency. The remaining sections of the paper are arranged as follows: section 2, presents the system model for the proposed algorithm. Section 3 displays the results of the simulation. Finally, in section 4 , the conclusion of the paper is presented.

\section{SYSTEM MODEL FOR THE PROPOSED ALGORITHM}

\subsection{SEH model for the WSNs}

Solar energy harvesting (SEH) is a technique that reuses unused solar energy from the ambient and transforms the light energy obtained into electrical energy. The light energy from sunlight rays should be completely used. The SEH-WSN node will use solar energy to operate the sensor node circuit and charge the battery for the future use of the sensor node. The energy harvested from solar radiation can be estimated on the basis of seasonal and diurnal cycles and weather prediction for the region. There are several predictive algorithms available to forecast potential available solar energy over time [24], [26], [27]. Sharma and Bhondekar [28], proposed the production of electricity from solar energy is unregulated, and it can be predicted for the majority of SEH sensor network scenarios. In the model, the energy harvested over time is assumed to be uniform for all nodes used when deploying WSN in an open environment. As shown in Figure 1, energy harvested is simulated at different times of the day, based on the difference in diurnal variations in solar intensity. Assuming the day is divided into $24 \mathrm{WSN}$ operating rounds, each round is of one-hour duration and the highest energy harvested during this round is $E h_{\max }$. The total energy-harvested Eh by node i in round $\mathrm{r}$ of WSN operations is given by [28].

$$
E h_{i}(r)=\left\{\begin{array}{lr}
0, & r \bmod 24 \leq 7 \\
\frac{E h_{\max }((r \bmod 24)-7)}{3}, & 7<r \bmod 24 \leq 10 \\
E h_{\max }(12-0.2(r \bmod 24)), & 10<r \bmod 24 \leq 15 \\
\frac{E h_{\max }(18-(r \bmod 24))}{3}, & 15<r \bmod 24 \leq 18 \\
0, & 18<r \bmod 24
\end{array}\right.
$$

The average energy-harvested by any node in where is between $(1 \leq \mathrm{i} \leq \mathrm{N})$ per round (hour) is given by: 
$E h_{a v}=\frac{E h_{\max }}{24}$

Random energy harvesting will be used in all nodes to simulate real energy harvesting, as shown in (3). The value of $\left(E h_{\max }\right)$ will be randomly adjusted over $\left(E h_{\text {lowest }}, E h_{\text {highest }}\right)$ for each node. Whereas, $E h_{\text {lowest }}$ represents the lowest value of energy harvested by the node depending on the location of the node and the surrounding weather conditions, while $E h_{\text {highest }}$ represents the highest value of energy harvested under ideal conditions.

$$
E h_{\text {max }}=E h_{\text {lowest }}+\frac{\left(E h_{\text {highest }}-E h_{\text {lowest }}\right)}{\left(1+p_{h}\right)}
$$

where $p_{h}$ represents the optimal harvest energy probability.

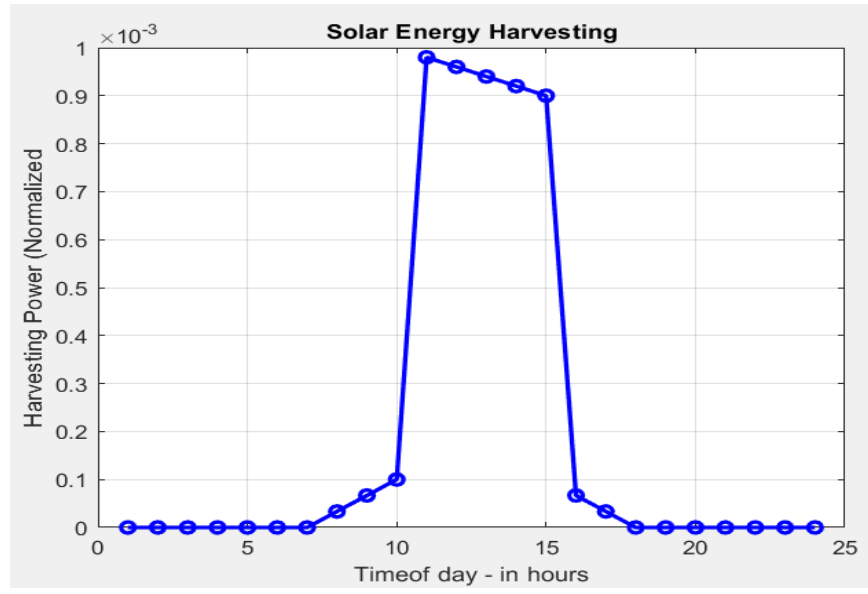

Figure 1. Simulation of a sensor node solar energy harvest process throughout the duration of a 24-hour day

\subsection{EHEAR algorithm}

\subsubsection{Network and wireless communication model}

Before going into the specifics of the algorithm, it's vital to establish the set of requirements and assumptions that the algorithm must meet.

- Assume $\mathrm{N}$ sensor nodes are randomly deployed across a $\mathrm{M} \times \mathrm{M}$ area.

- After deployment, the sensor nodes are in a fixed position, and each node has only one unique ID.

- There is only one BS located in the center of the area, which is in stationary mode and has a continuous power supply.

- The Node has characteristics such as energy heterogeneous, solar energy acquisition and data fusion.

- The communication link is symmetrical, and it can determine the distance between the sender and itself based on the sender's transmitting power.

- The transmitting power of the node can be adjusted according on the distance. When no data has to be transferred, the node enters a sleeping mode to conserve energy.

- The sensor nodes will communicate with each other and with the BS through single-hop communication.

- The clustering function is dynamic, which means, as soon as the clusters are formed and/or cluster heads are selected, they will be changeable according to certain thresholds.

- The new round will start based on a time condition that is set at 1 hour (60 minutes), which is related to the time needed for the node to harvest the energy from the solar system every hour.

The simple radio communication model proposed by [29] was used in this paper to demonstrate energy dissipation in the transmitter (Tx) to operate the radio electronics and the power amplifier, and in the receiver $(\mathrm{Rx})$ to operate the radio electronics, as shown in Figure 2. The energy consumed by an active node $i$ during sending a message of length $l_{m}$ bits across a distance $d$ can be calculated as (4),

$$
E_{T x}\left(l_{m}, d\right)=\left\{\begin{array}{l}
l_{m} E_{\text {elec }}+l_{m} \varepsilon_{f s} d^{2} \text { if } d \leq d_{o} \\
l_{m} E_{\text {elec }}+l_{m} \varepsilon_{m p} d^{4} \text { if } d>d_{o}
\end{array}\right.
$$


where $E_{\text {elec }}$ is the energy dissipation per bit of a transmitter Tx/receiver Rx electronic circuit. $\varepsilon_{f s}$ and $\varepsilon_{m p}$ are the radio parameter associated with energy dissipation in $T x$ amplifiers in free space and multipath scenarios, respectively (when $d_{0}=\sqrt{\frac{\varepsilon_{f s}}{\varepsilon_{m p}}}$ ). In the receiver side, the energy consumption is based on the message size that is transmitted from the active cluster member CM nodes.

$$
E_{R x}\left(l_{m}, d\right)=l_{m} E_{\text {elec }}
$$

where $E_{R x}$ is the dissipating energy to receive the $l_{m}$-bits message.

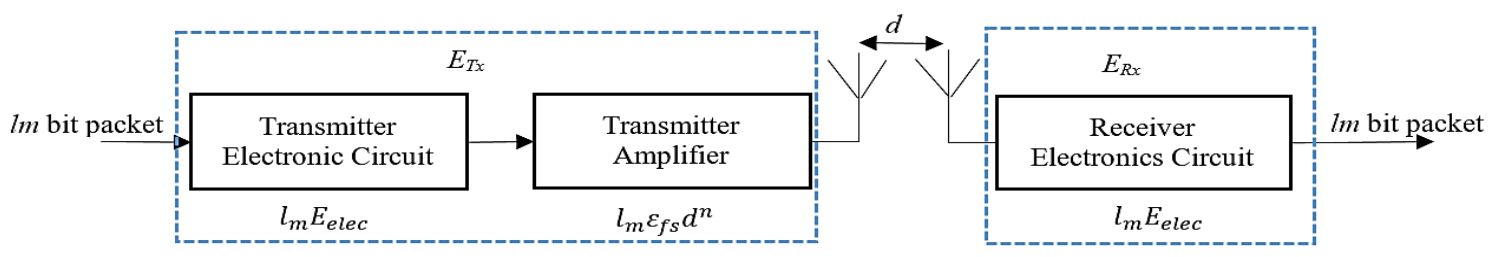

Figure 2. Wireless communication radio model

\subsubsection{The clustering function}

The main goal of the clustering function is to optimize the energy consumption of the sensor nodes. This will be achieved by managing and controlling the process of cluster head selection and cluster formation. In general, the clustering function is separated into two main phases: the setup phase, where the cluster head nodes are selected, and cluster formation. The steady state phase is when the organized nodes begin to communicate with their cluster heads. The idea for the proposed algorithm is illustrated in Figure 3(a) (see Appendix). The sections that follow will go over these two phases of the proposed algorithm in detail.

a) Setup phase

According to the proposed model, the probable cluster head $\mathrm{CH}$ will be selected as well as the cluster that will be formed based on the highest residual energy of the node, its distance from the BS, and the distance between the sensor and its neighbor. The remaining sensors will be considered as cluster members CMs and will join with the nearest cluster head as shown in Figure 3(b) (see Appendix). The sensor S(i) will be considered as qualified to be chosen as $\mathrm{CH}$, if the following conditions are met: first, if the distance between the S(i) node and its neighbor is less than 3 meters, second, if the distance between it and the BS $d_{S t o B S}(i)$ is greater than $(\mathrm{d} 2)$ and less than $(\mathrm{d} 0)$ and finally, if the residual energy level of the sensor $\mathrm{S}(\mathrm{i})$ is greater than the first threshold Th1, else S(i) will be considered a cluster member CM. The pseudocode used to set the first threshold Th1 is presented in algorithm 1 Table 1. According to the pseudocodes Algorithm 1, the $E_{S}(i)$ represent the residual energy of sensor node $S(i)$ for $i=1,2, \ldots, \mathrm{N}$. The matrix laboratory (MATLAB) function (maxk) returns a vector containing the N/2 largest elements of $E_{S}(i)$. Then will set first threshold Th1 equal to the lowest value of $E_{\max } . N_{C H}$ represents the number of cluster heads and $N_{C M}$ represents the number of cluster members. Therefore, the total number of nodes $N$ for both cases will be as illustrated (6);

$$
N=N_{C H}+N_{C M}
$$

With regard to managing the sensor node's energy utilization, and after completing the clustering function, the active-sleep mechanism will be adopted. The $\mathrm{CH}$ nodes and $\mathrm{CM}$ nodes will be divided into active and sleeping based on their residual energy and the required number of active cluster head nodes. Based on design assumptions, the number of active cluster head nodes and sleep cluster head nodes will be set equal to $1 / \mathrm{P}$. The $\mathrm{CH}$ nodes will be divided into active cluster head nodes $\mathrm{N}_{\mathrm{CHA}}$ and sleep cluster head nodes $\mathrm{N}_{\mathrm{CHS}}$ based on their residual energy according to the second threshold value Th2. Also, for the cluster member nodes, they will be divided into active cluster members $\mathrm{N}_{\mathrm{CMA}}$, and sleep cluster members $\mathrm{N}_{\mathrm{CMS}}$ according to the third threshold Th3 values as shown in Figure 3(c) (see Appendix). If the sensor S(i) which is selected as $\mathrm{CH}$ has a residual energy level greater than the second threshold $T h 2$, it will be considered as an active $\mathrm{CH}$; otherwise, it will go into sleep mode. In terms of cluster members, if the sensor S(i) chosen as a $\mathrm{CM}$ has a residual energy level greater than the third threshold $T h 3$, but less than $T h 2$, it will be set to an active CM; otherwise, it will enter sleep mode. In order to understand this step, the pseudocode for setting the value of Th2, Th3 and active/sleep cluster heads and cluster members is presented in Algorithm 2 Table 1. In general, the total number of cluster head nodes $N_{C H}$ in both cases is illustrated (7). 


$$
N_{C H}=N_{C H A}+N_{C H S}
$$

Furthermore, the total number of cluster member nodes $N_{C M}$ is represented (8).

$$
N_{C M}=N_{\mathrm{CMA}}+N_{\mathrm{CMS}}
$$

Finally, the total number of active nodes $\left(N_{\text {ACTIVE }}\right)$ (as active $\mathrm{CHs}$ and $\mathrm{CMs}$ ) is explained in the (9).

$$
N_{\text {ACTIVE }}=N_{C H A}+N_{C M A}
$$

\begin{tabular}{|c|c|c|}
\hline \multicolumn{2}{|r|}{ Algorithm 1} & Algorithm 2 \\
\hline$>$ & {$\left[E_{\max }\right]=\operatorname{maxk}\left(E_{S}(i), \mathrm{N} / 2\right)$} & {$[\mathrm{ECH} \max ]=\operatorname{maxk}\left(E_{C H}, 1 / p\right)$} \\
\hline$>$ & Set Th1 $1=E_{\max }(\mathrm{N} / 2)$ & Set Th2 $=$ ECHmax $(1 / p)$ \\
\hline$>$ & processing group 2 & {$[\mathrm{ECMmax}]=\operatorname{maxk}\left(E_{C M}, 1 / \alpha\right)$} \\
\hline & & Set Th3 $=$ ECMmax $(1 / \alpha)$ \\
\hline & & processing group 3 \\
\hline
\end{tabular}

Table 1. The algorithm $1 \& 2$ for setting the threshold levels

- Energy consumption analysis for clustering function

In order to understand how the nodes consumption and manage their energy, this section includes the energy consumption model for the proposed design. For cluster head nodes, if there are $N_{\mathrm{CHA}}$ clusters, there are on average $N_{A C T I V E} / N_{\mathrm{CHA}}$ nodes per cluster (one active $\mathrm{CH}$ and $\left(\left(N_{\text {ACTIVE }} / N_{\mathrm{CHA}}\right)-1\right)$ active CM nodes). The energy dissipated $E_{C H A}$ in the active $\mathrm{CH}$ node during a single round in various fields is:

$$
E_{C H A}=\left(\frac{N_{A C T I V E}}{N_{C H A}}-1\right) E_{R x C H A}+\left(\frac{N_{A C T I V E}}{N_{C H A}}\right) E_{D A}+E_{T X C H A}
$$

where $\left(E_{R x C H A}=l_{m} E_{\text {elec }}\right)$ is the dissipating energy to receive the lm-bits message from the active cluster member nodes. During the aggregation of data from active CM nodes, the energy dissipated to aggregate data $E_{D A}$ can be expressed as $\left(E_{D A}=l_{m} E_{d a}\right)$. The dissipating energy to transmit the aggregate data from the active $\mathrm{CH}$ to the $\mathrm{BS}$ is $E_{T x C H A}$. Assuming the base station BS in the central of the target area and that the distance of any node to the BS is less than $d 0$. So the free space model ( $d^{2}$ power loss ) will be recommended for the transmissions side so $\left(E_{T x C H A}=l_{m} E_{e l e c}+l_{m} \varepsilon_{f S} d_{C H_{-} t o \_B S}^{2}\right)$.

$$
E_{\text {CHA }}=\left(\frac{N_{\text {ACTIVE }}}{N_{\text {CHA }}}\right) l_{m} E_{\text {elec }}+\left(\frac{N_{\text {ACTIVE }}}{N_{\text {CHA }}}\right) l_{m} E_{\text {da }}+l_{m} E_{\text {elec }}+l_{m} \varepsilon_{f s} d_{\text {CHA_to_BS }}^{2}
$$

On other hand, for active cluster member node only needs to send its data to the corresponding active $\mathrm{CH}$ once in a frame. The distance to the active cluster head is typically less than $\mathrm{d} 0$, so the energy dissipation $E_{C M A}$ in each active cluster member node is:

$$
E_{C M A}=l_{m} E_{\text {elec }}+l_{m} \varepsilon_{f s} d_{C M A_{-} t o_{-} C H A}^{2}
$$

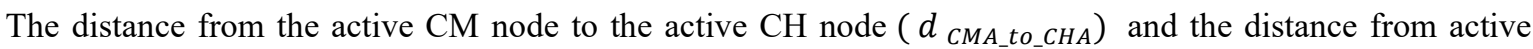
$\mathrm{CH}$ to the BS ( $\left.d_{\text {CHA_to BS }}\right)$ for scenario of equal node distribution [28] with NCHA the number of clusters are defined as $\quad\left(d_{C M A_{-} t o_{-} C H A}=d 2=\frac{M}{\sqrt{2 \pi N_{a C H}}}\right)$ and $\left(d_{C H A_{-} t o_{-} B S}=d 1=0.765 \frac{M}{2}\right)$ where $\mathrm{M}$ represents the sensing area.

b) Steady state phase

At this phase, the sensor nodes will start sensing and transmitting data to the active cluster head nodes once every cycle, according to a timer. The active $\mathrm{CH}$ nodes, will collect data from their members, aggregate it, and send it to the base station. The main concept for this step is shown in Figure 3(d) (see Appendix). Overall, The energy dissipated in a cluster $E_{\text {cluster }}$ during the frame is:

$$
E_{\text {cluster }}=E_{C H A}+\left(\frac{N_{A C T I V E}}{N_{C H A}}-1\right) E_{C M A} \approx E_{C H A}+\frac{N_{A C T I V E}}{N_{C H A}} E_{C M A}
$$

Finally, the total energy consumption for the whole network is shown in the (14).

$$
E_{\text {total }}=N_{\mathrm{CHA}} E_{\text {cluster }}=N_{\mathrm{CHA}} E_{A C H}+N_{\text {ACTIVE }} E_{A C M}
$$


In the case of nodes with energy-harvesting capability and an energy heterogeneity situation, a round's effective energy will take into account both lost and harvested energy. According the recommended solar energy harvesting technique defined in section 2.1, the average harvested energy Ehavg can be considered as the collecting energy per round for any node. The effective energy dissipation with the average harvested energy of the network for one round of operation is given by:

$$
E_{\text {Round }}=E_{\text {total }}-E_{\text {hav }}
$$

\section{SIMULATION AND RESULTS}

In our simulation, 100 sensor nodes with solar energy harvesting are randomly distributed in a 100x100 m area, with the base station located at $(50 \mathrm{~m}, 50 \mathrm{~m})$ as shown in Figure 4. Assume the sensors energy Es are randomly set from (0.5-1J). MATLAB was used to run the simulation and analysis of the proposed model, and Table 2 lists the parameters that were taken into consideration. As mentioned previously, during the setup phase, the $\mathrm{CHs}$ will be selected based on distance and residual energy to extend the network's life. In addition to that, the nodes will be classifying into active and sleep groups. This classification is to ensure that the node has enough energy to deliver information to the BS and prolong the life of the network. It is worth noting that the entry of a number of nodes into a state of early sleep, despite the presence of sufficient energy to perform their work for several rounds, contributed to the strengthening of the network's work, as the node will not start sleeping in a state of zero energy as in the rest of the algorithms. For energy heterogeneity consideration, low energy adaptive clustering hierarchy (LEACH), distributed energy efficient clustering (DEEC), and stable election protocol (SEP) are upgraded as discussed in [28], [30]. All three algorithms have been upgraded to include single-hop communication and random level energy-harvesting capabilities as discussed in section 2. Uniformity in the energy-harvesting behavior of these algorithms was maintained to provide an unbiased comparison of observing different cluster header selection behaviors and mechanisms for different algorithms. As shown in Figure 5, you will notice that there are 10 active $\mathrm{CH}$ nodes and 10 sleeping $\mathrm{CH}$ nodes. With this, there will be 10 clusters distributed around the BS to cover the target area. Each cluster contains from 5 to 8 active CM nodes according to the location of the active $\mathrm{CH}$ during the round (r). According to the residual energy, the number of sleep CM nodes $\alpha$ will be semi-constant here, which is equal to ( 9 or 10$)$ nodes, as shown in Figure 5. The remaining CM nodes will be active based on the amount of energy they have available during the round (r).

Table 2. Simulation parameters

\begin{tabular}{|c|c|c|c|c|c|}
\hline Simulation parameter & Symbol & Value & Simulation parameter & Symbol & Value \\
\hline Number of sensor nodes & $\mathrm{N}$ & 100 & Data Aggregation energy consumption & $\mathrm{E}_{\mathrm{DA}}$ & $5 \mathrm{~nJ} / \mathrm{bit} / \mathrm{signal}$ \\
\hline Deployment area & M & $100 \times 100 \mathrm{~m}$ & Heterogeneous initial energy & Es & Rand $(0.5,1) \mathrm{J}$ \\
\hline Total number of rounds & $\mathrm{r}$ & 8760 hours & Desired probability of $\mathrm{CH}$ & $P$ & 0.1 \\
\hline Packet length & $m l$ & 4000 bit & Distance between active $\mathrm{CH}$ and $\mathrm{BS}$ & $d 1$ & $38.25 \mathrm{~m}$ \\
\hline Sink node position & - & $50 \times 50$ & Distance between active CMs and $\mathrm{CHs}$ & $d 2$ & $24.9649 \mathrm{~m}$ \\
\hline Network deployment & - & Randomly & Threshold to receive a message of $\mathrm{ml}$ bit & $d 0$ & $87.7058 \mathrm{~m}$ \\
\hline $\begin{array}{l}\text { Energy consumption of } \mathrm{Tx} R \mathrm{R} \\
\text { electronics circuit }\end{array}$ & $E_{\text {elec }}$ & $50 \mathrm{~nJ} / \mathrm{bit}$ & Harvested Energy lower bound & $E h_{\text {lowest }}$ & $0.00005 \mathrm{~J}$ \\
\hline Free-space channel parameter & $\mathrm{E}_{\mathrm{fs}}$ & $10 \mathrm{pJ} / \mathrm{bit} / \mathrm{m} 2$ & Harvested Energy upper bound & Eh $h_{\text {highest }}$ & $0.00015 \mathrm{~J}$ \\
\hline Multipath channel parameter & $\mathrm{E}_{\mathrm{mp}}$ & $\begin{array}{c}0.0013 \\
\mathrm{pJ} / \mathrm{bit} / \mathrm{m} 4\end{array}$ & Optimal harvest energy probability & $p_{h}$ & Rand $(0,1)$ \\
\hline
\end{tabular}

As shown in Figure 6 and Figure 7, the number of active/sleep nodes affecting the longevity of the network. For the EH-SEP the first node enters sleep mode in 1912th round, the number of sleep nodes progressively increases until it reaches 42 in the 8760th round. The first node to enter the sleep mode in the EH-DEEC algorithm was in the 5373rd round, and the number of nodes that entered the sleep mode gradually increased to become in the 8760th round's 52 nodes. The first node in the EH-LEACH algorithm entered sleep mode in the 5071 st round, and the number of nodes increased until it reached 58 sleeping nodes in the 8760th round as seen in Table 3 and Figure 7. It is clear that our proposed algorithm EHEARA is more efficient and more stable than other algorithms. The simulation results also demonstrate the algorithm's stability over the working period, where the number of sleeping nodes settled 19 nodes with better performance in terms of packets sent to BS and energy consumption as shown in Figures 8 and 9 and Table 3 .

As shown in Figure 8 and Table 3, when the proposed algorithm is used, the energy of the network after the 8760 th round is equal to $35.5355 \mathrm{~J}$. These results are obtained because the proposed algorithm was computed in the most accurate and balanced manner, and total energy is utilized in a more balanced and distributed manner while $\mathrm{CH}$ is selected, where the proposed algorithm beats other existing algorithms. The 
simulation results for other algorithms (LEACH, DEES, SEP), show that the network energy has reached its lowest level of less than 8 joules in round 8760. In this regard, the proposed algorithm EHEARA increases the network lifetime over longer rounds better than the three clustering algorithms. Figure 9 shows the number of packets sent to the BS, as well as how the proposed algorithm increase network throughput. Table 3, shows the results of the simulation and comparison between the proposed algorithm and the rest of the algorithms in terms of residual energy, number of sent packets and number of active nodes.

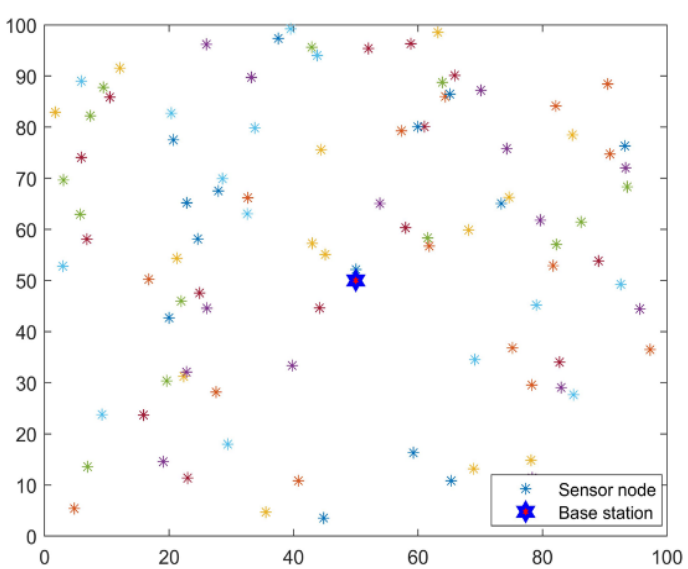

Figure 4. Sensor nodes with BS

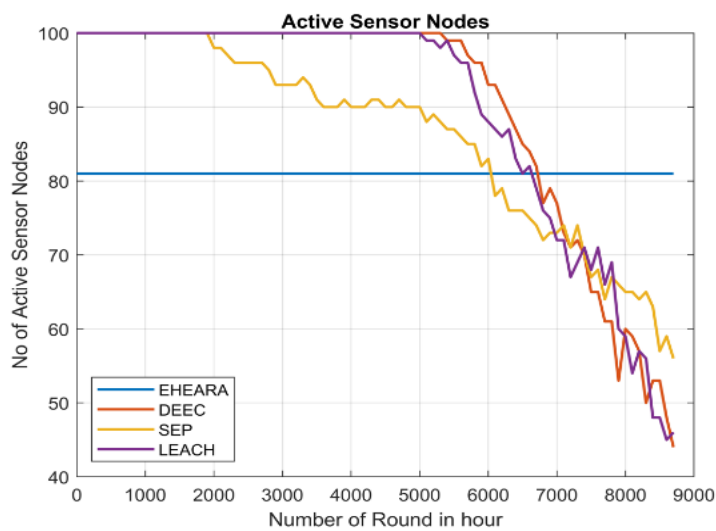

Figure 6. Comparison of number of active nodes

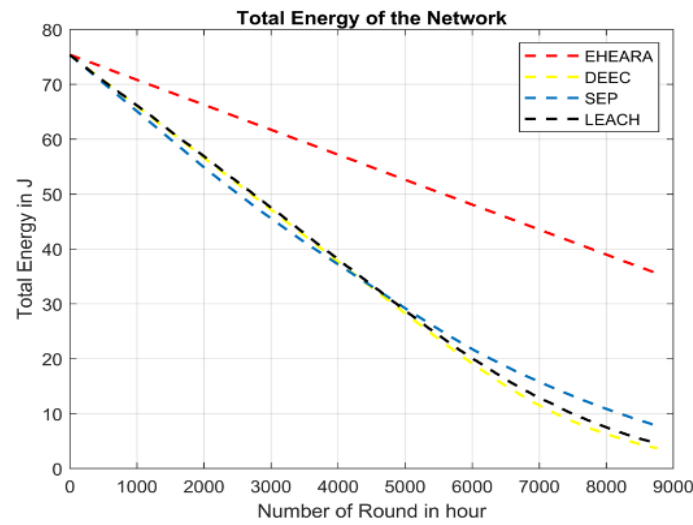

Figure 8. Comparison of total residual energy at round $r$

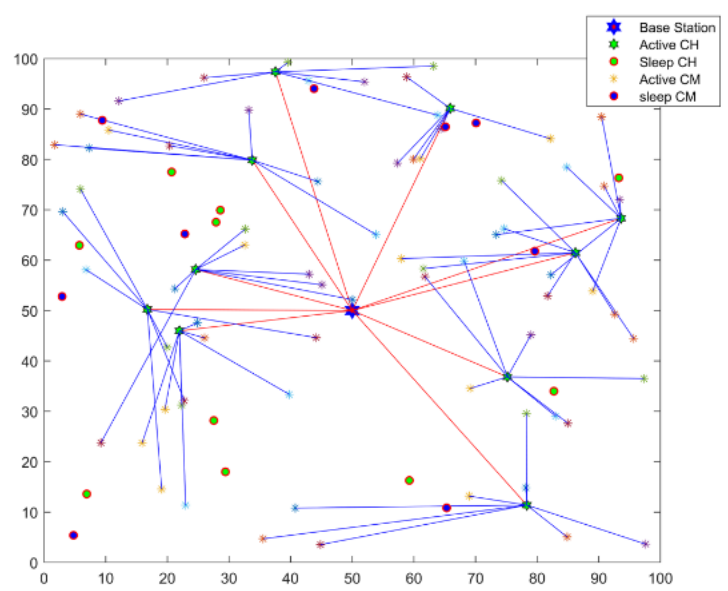

Figure 5. Cluster formation with active/sleep nodes

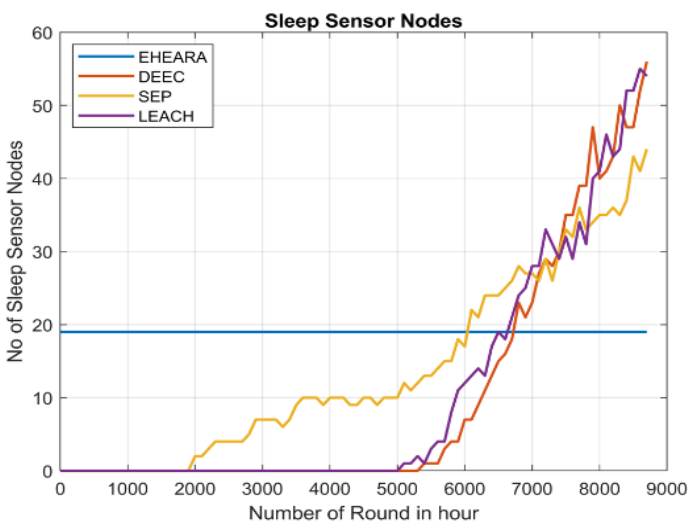

Figure 7. Comparison of number of sleeping nodes

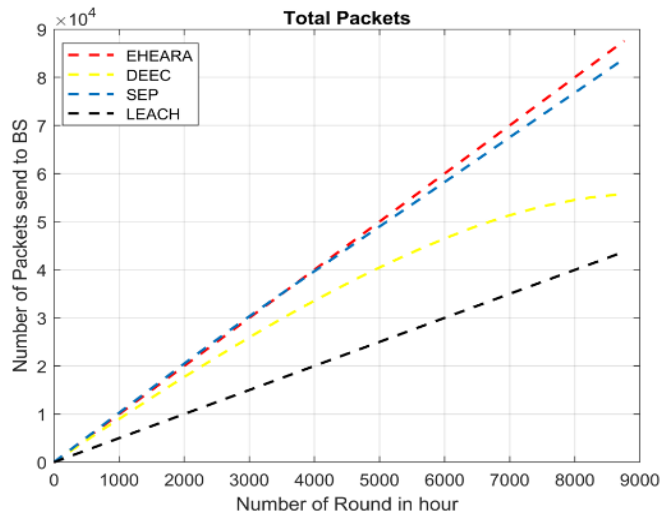

Figure 9. Comparison of the number of packets sent to the BS 
Table 3. Compared with other algorithms

\begin{tabular}{|c|c|c|c|c|c|c|c|c|c|c|c|c|}
\hline \multirow{2}{*}{$\begin{array}{l}\text { No. of } \\
\text { round }\end{array}$} & \multicolumn{4}{|c|}{ Network residual energy in $\mathrm{J}$} & \multicolumn{4}{|c|}{ Total number of packets send to BS } & \multicolumn{4}{|c|}{ Number of sensor nodes active per $r$} \\
\hline & EHEARA & DEEC & SEP & LEACH & EHEARA & DEEC & SEP & LEACH & EHEARA & DEEC & SEP & LEACH \\
\hline 1000 & 70.8114 & 66.0254 & 65.0973 & 66.1661 & 9990 & 8961 & 10252 & 5000 & 81 & 100 & 100 & 100 \\
\hline 3000 & 61.7266 & 47.1466 & 45.5679 & 47.5222 & 29990 & 25936 & 30308 & 15000 & 81 & 100 & 93 & 100 \\
\hline 4000 & 57.1860 & 37.6928 & 37.2031 & 38.1029 & 39990 & 33538 & 39696 & 20000 & 81 & 100 & 90 & 100 \\
\hline 5000 & 52.6017 & 28.2690 & 29.2091 & 28.7995 & 49990 & 40455 & 48979 & 25000 & 81 & 100 & 90 & 100 \\
\hline 8000 & 38.9920 & 6.3001 & 10.8748 & 7.5234 & 79990 & 54509 & 76791 & 39967 & 81 & 59 & 63 & 59 \\
\hline 8760 & 35.5355 & 3.7107 & 7.7921 & 4.6184 & 87590 & 55597 & 83881 & 43755 & 81 & 48 & 58 & 42 \\
\hline
\end{tabular}

\section{CONCLUSION}

WSN faces many obstacles to being the ideal solution for environmental monitoring. One of the biggest obstacles was power consumption and changing the batteries. Therefore, using solar energy harvesting is considered one of the effective solutions, but we need to balance the time it needed to recharge the node battery with the time it needed to consume the harvested energy to ensure a steady-state performance for the network. In this paper, we prposed a new algorithm that takes into consideration this required balance and ensures to keep the network working with the same performance it starts with for a long time. The proposed algorithm offers the largest amount of residual energy compared to other algorithms. In addition, it is the best performer in terms of the number of packets sent to BS. The proposed algorithm also maintains the same performance throughout all 8760 rounds representing an entire year, by working with 81 active sensor nodes and 19 sleeping nodes through all 8760 rounds.

\section{APPENDIX}

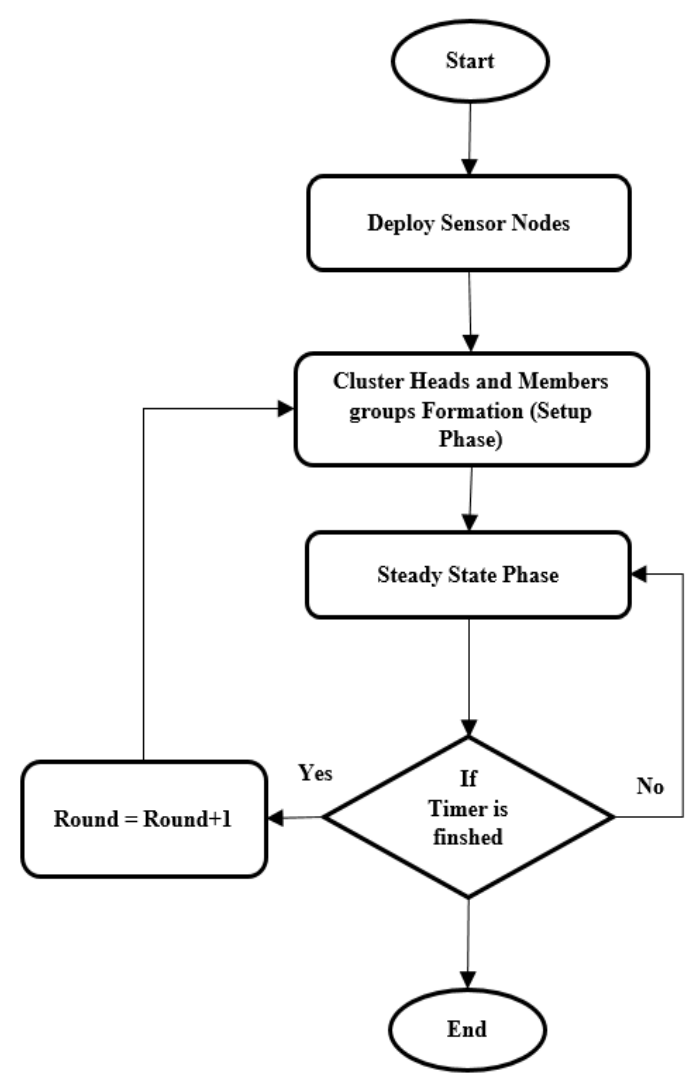

(a)

Figure 3. Flowchart of proposed algorithm: (a) the main flowchart of proposed algorithm 


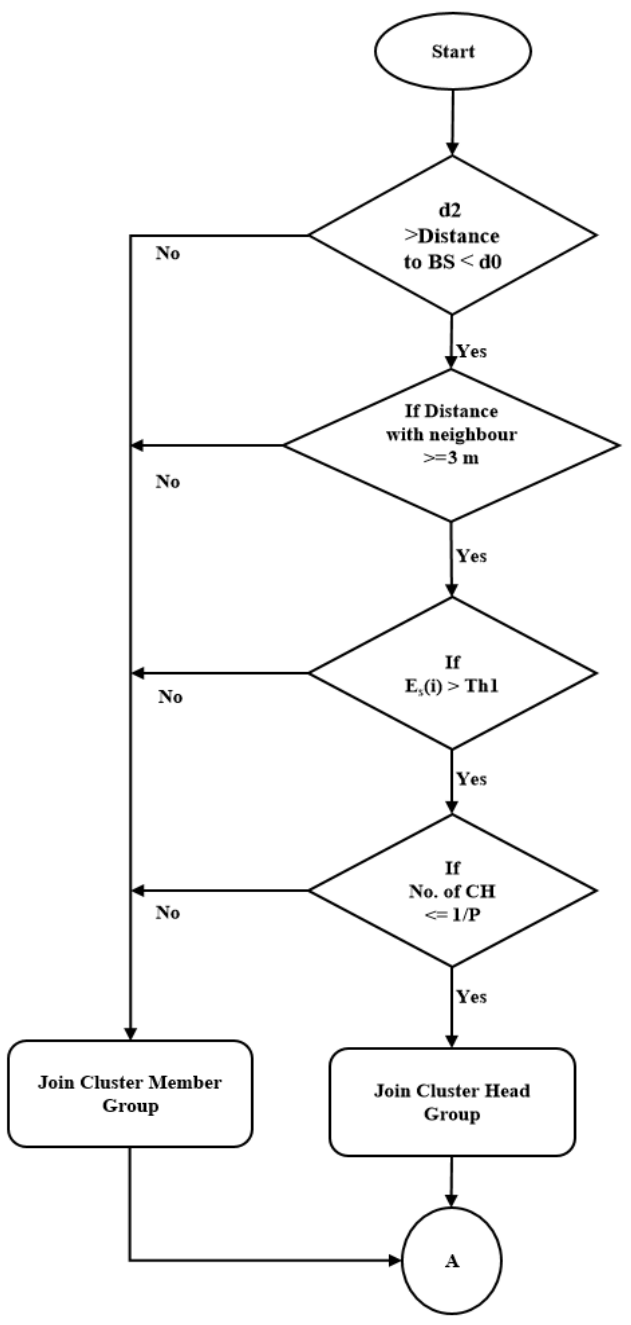

(b)

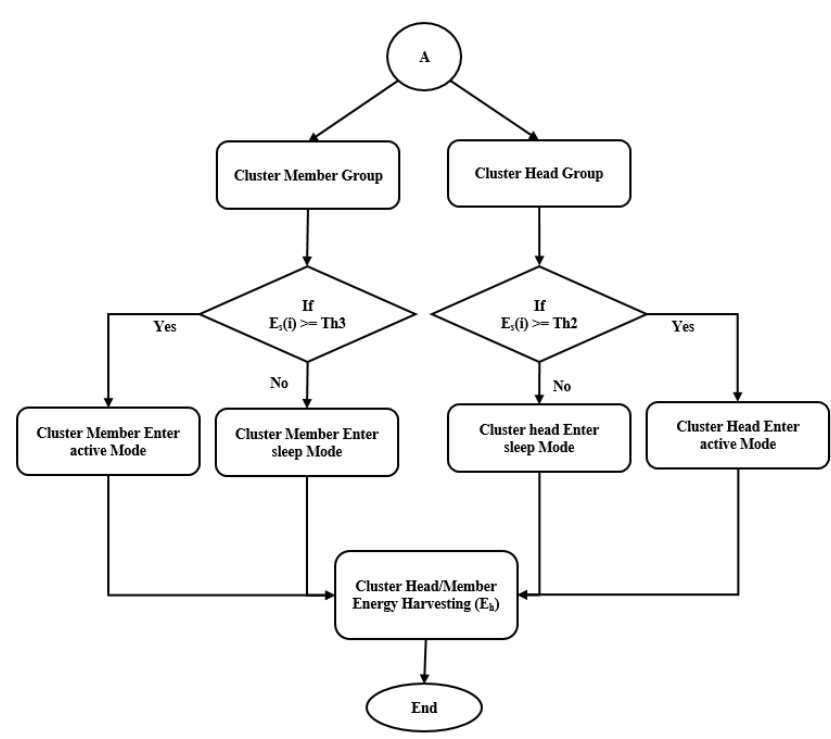

(c)

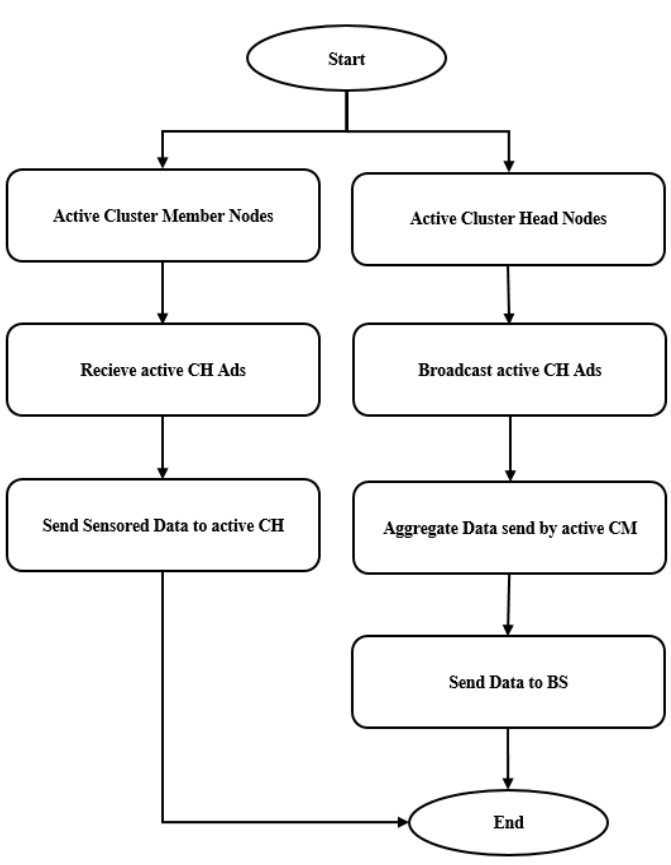

(d)

Figure 3. Flowchart of proposed algorithm: (b) setup phase (cluster head and cluster member groups formation), (c) setup phase (cluster head/member active/sleep mode) and (d) steady state phase (continue) 


\section{REFERENCES}

[1] J. Y. Lee and D. Lee, "Improvement of CH election in three-level heterogeneous WSN," Indonesian Journal of Electrical Engineering and Computer Science, vol. 13, no. 1, pp. 272-278, 2019, doi: 10.11591/ijeecs.v13.i1.pp272-278.

[2] M. M. Saleh, A. A. Abbas, and A. Hammoodi, "5G cognitive radio system design with new algorithm asynchronous spectrum sensing," Bulletin of Electrical Engineering and Informatics, vol. 10, no. 4, pp. 2046-54, Aug. 2021, doi: 10.11591/eei.v10i4.2839.

[3] B. Abood and Y. K. Al-Rikabi, "Lifetime enhancement for clustering protocols in heterogeneous wireless sensor networks," Indonesian Journal of Electrical Engineering and Computer Science, vol. 14, no. 3, pp. 1305-1314, 2019, doi: 10.11591/ijeecs.v14.i3.pp1305-1314.

[4] B. S. Jayasri and G. Raghavendra Rao, "A novel predictive optimization scheme for energy-efficient reliable operation of a sensor in dynamic scenarios," International Journal of Electrical and Computer Engineering, vol. 10, no. 1, pp. 323-332, 2020, doi: 10.11591/ijece.v10il.pp323-332.

[5] A. Naushad, G. Abbas, S. A. Shah, and Z. H. Abbas, "Energy Efficient Clustering with Reliable and Load-Balanced Multipath Routing for WSNs," 2020 3rd International Conference on Advancements in Computational Sciences (ICACS), 2020, doi: 10.1109/ICACS47775.2020.9055957.

[6] M. Q. Taha, Z. H. Ali, and A. K. Ahmed, "Two-level scheduling scheme for integrated 4G-WLAN network," International Journal of Electrical and Computer Engineering, vol. 10, no. 3, pp. 2633-2643, 2020, doi: 10.11591/ijece.v10i3.pp2633-2643.

[7] M. Saleh, "WSNs and IoT Their Challenges and applications for Healthcare and Agriculture: A Survey," Iraqi J. Electr. Electron. Eng., vol. sceeer, no. 3d, pp. 37-43, Jul. 2020, doi: 10.37917/ijeee.sceeer.3rd.6.

[8] A. K. Ahmed, M. Q. Taha, and A. S. Mustafa, "On-road automobile license plate recognition using co-occurrence matrix," Journal of Advanced Research in Dynamical and Control Systems, vol. 10, no. 7, pp. 387-393, 2018.

[9] L. K. Ketshabetswe, A. M. Zungeru, M. Mangwala, J. M. Chuma, and B. Sigweni, "Communication protocols for wireless sensor networks: A survey and comparison," Heliyon, vol. 5, no. 5, 2019, doi: 10.1016/j.heliyon.2019.e01591.

[10] B. Hasan, S. Alani, and M. A. Saad, "Secured node detection technique based on artificial neural network for wireless sensor network," Int. J. Electr. Comput. Eng., vol. 11, no. 1, 2021, doi: 10.11591/ijece.v11i1.pp536-544.

[11] Y. S. Alwan, M. S. Zidan, and M. Q. Taha, "Evaluation of mobile microwave electric field severity at al-door residential complex in Iraq," International Journal of Electrical and Computer Engineering, vol. 14, no. 3, pp. 1281-1285, 2019, doi: 10.11591/ijeecs.v14.i3.pp1281-1285.

[12] M. Zeng, X. Huang, B. Zheng, and X. Fan, "A heterogeneous energy wireless sensor network clustering protocol," Wireless Communications and Mobile Computing, vol. 2019, 2019, pp. 1-11, doi: 10.1155/2019/7367281.

[13] K. Nagarathna, "Energy-aware strategy for data forwarding in IoT ecosystem," International Journal of Electrical and Computer Engineering, vol. 10, no. 5, pp. 4863-4871, 2020, doi: 10.11591/ijece.v10i5.pp4863-4871.

[14] V. Sivasankarareddy and G. Sundari, "Survey on wireless sensor networks: Energy efficient optimization routing algorithms," Indonesian Journal of Electrical Engineering and Computer Science, vol. 19, no. 2, pp. 756-765, 2020, doi: 10.11591/ijeecs.v19.i2.pp756-765.

[15] R. Sridhar and N. Guruprasad, "Energy efficient chaotic whale optimization technique for data gathering in wireless sensor network," International Journal of Electrical and Computer Engineering, vol. 10, no. 4, pp. 4176-4188, 2020, doi: 10.11591/ijece.v10i4.pp4176-4188.

[16] N. M. S. Almurisi and T. Srinivasulu, "A novel scheme for energy-efficient bridge layer in sensor-cloud," Indonesian Journal of Electrical Engineering and Computer Science, vol. 18, no. 2, pp. 1048-1056, 2020, doi: 10.11591/ijeecs.v18.i2.pp1048-1056.

[17] D. L. Shanthi and K. Prasanna, "Energy efficient intelligent routing in WSN using dominant genetic algorithm," I International Journal of Electrical and Computer Engineering, vol. 10, no. 1, pp. 500-511, 2020, doi: 10.11591/ijece.v10i1.pp500-511.

[18] J. Metan and K. N. N. Murthy, "FSDA: Framework for Secure Data Aggregation in Wireless Sensor Network for Enhancing Key Management," International Journal of Electrical and Computer Engineering, vol. 8, no. 6, pp. 4684-4692, 2018, doi: 10.11591/ijece.v8i6.pp4684-4692.

[19] A. El Aalaoui and A. Hajraoui, "Energy efficiency of organized cluster election method in wireless sensor networks," Indonesian Journal of Electrical Engineering and Computer Science, vol. 18, no. 1, pp. 218-226, 2019, doi: 10.11591/ijeecs.v18.i1.pp218-226.

[20] D. Ye and M. Z. Au, "A Self-Adaptive Sleep/Wake-Up Scheduling Approach for Wireless Sensor Networks," IEEE Transactions on Cybernetics, vol. 48, no. 3, pp. 979-992, 2018, doi: 10.1109/TCYB.2017.2669996.

[21] D. Gurusamy and S. Abas, "Modified clustering algorithms for energy harvesting wireless sensor networks- a survey," 2020 21st International Arab Conference on Information Technology (ACIT), 2020, doi: 10.1109/ACIT50332.2020.9300078.

[22] A. Kumar and B. Alam, "Energy harvesting earliest deadline first scheduling algorithm for increasing lifetime of real time systems," International Journal of Electrical and Computer Engineering, vol. 9, no. 1, pp. 539-545, 2019, doi: 10.11591/ijece.v9i1.pp539-545.

[23] S. Pundir, M. Wazid, A. Bakshi, and D. P. Singh, "Optimized low-energy adaptive clustering hierarchy in wireless sensor network," Advances in Intelligent Systems and Computing, vol. 1162, pp. 34-42, 2021, doi: 10.1007/978-981-15-4851-2_4.

[24] T. Lu, G. Liu, and S. Chang, "Energy-efficient data sensing and routing in unreliable energy-harvesting wireless sensor network," Wireless Networks, vol. 24, no. 2, pp. 1-15, 2018, doi: 10.1007/s11276-016-1360-6. 
[25] D. Sharma and A. P. Bhondekar, "Traffic and Energy Aware Routing for Heterogeneous Wireless Sensor Networks," IEEE Communications Letters, vol. 22, no. 8, pp. 1608-1611, 2018, doi: 10.1109/LCOMM.2018.2841911.

[26] O. M. Bushnaq, A. Chaaban, S. P. Chepuri, G. Leus, and T. Y. Al-Naffouri, "Sensor placement and resource allocation for energy harvesting IoT networks,” Digital Signal Processing, vol. 105, 2020, doi: 10.1016/j.dsp.2020.102659.

[27] A. Rashid, F. Khan, T. Gul, S. Khan, and F. K. Khalil, "Improving energy conservation in wireless sensor networks using energy harvesting system," International Journal of Advanced Computer Science and Applications (IJACSA), vol. 9, no. 1, 2018, doi: 10.14569/IJACSA.2018.090149.

[28] D. Sharma and A. P. Bhondekar, "An Improved Cluster Head Selection in Routing for Solar Energy-Harvesting Multi-heterogeneous Wireless Sensor Networks," Wirel. Pers. Commun., vol. 108, no. 4, 2019, doi: 10.1007/s11277-019-06518-4.

[29] W. B. Heinzelman, A. P. Chandrakasan, and H. Balakrishnan, "An application-specific protocol architecture for wireless microsensor networks," Wireless Personal Communications, vol. 1, no. 4, 2002, doi: 10.1109/TWC.2002.804190.

[30] X. N. Xu, M. B. Xiao, and W. Yan, "Clustering Routing Algorithm for Heterogeneous WSN with Energy Harvesting," Applied Mechanics and Materials, vol. 733, pp. 734-739, 2015, doi: 10.4028/www.scientific.net/amm.733.734.

\section{BIOGRAPHIES OF AUTHORS}
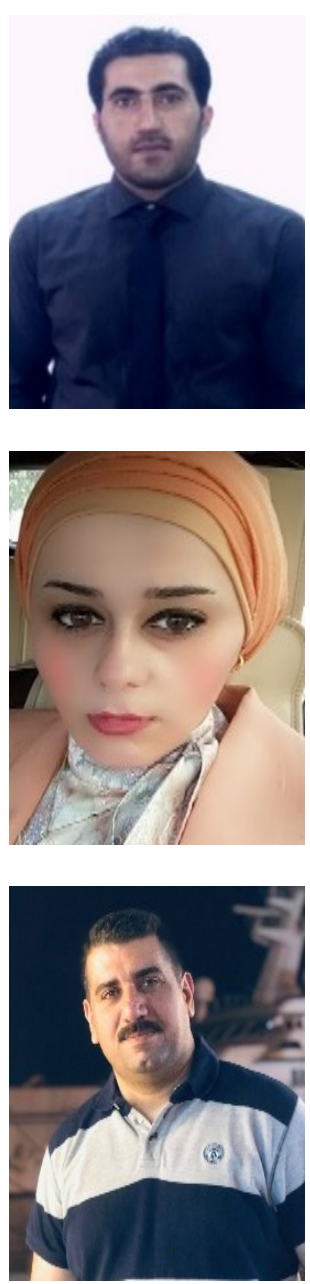

Mohammed Mehdi Saleh received a bachelor's degree in Electrical Engineering from the University of Anbar, Iraq in 2009 and a master's degree in Electronics and Communication Engineering (SSP) from Osmania University, India in 2014. He has served as a teaching assistant for the following undergraduate courses taught at Al-maaref University College: coding theory, computer network, real time signal processing, OFC and he is presently teaching staff at University of Anbar. His main interests are in 5G, WSNs.

Ruslan Saad Abdulrahman received her B.Sc. degree in Computer Engineering from Al-Nahrain University, Iraq (2003), and her M.Sc. in Computer Engineering from Al-Nahrain University/Iraq (2006). PhD in Computer Networks/University of Salford/UK (2017). She has served as a lecturer for the following undergraduate and postgraduate courses at Al-Nahrain University: computer networks, information technology, website design, wireless networks, multimedia communications, and operating systems. Her main interests are in WSNs, computer networks, and IoT.

Aymen Jaber Salman holds a B.Sc. in Electrical and Electronics Engineering and an M.Sc. in Computer Engineering and Information Technology from the University of Technology, Iraq, in 2002 and 2006, respectively. He received his Ph.D. in data Telecommunication and Networks from the University of Salford, UK, in 2017. Currently, he is the head of the Computer Engineering Department at Al-Nahrain University. His research interests include domain specific languages, model-driven development, web services, the internet of things (IoT) and wireless sensor networks. 